\title{
First Results of the Daya Bay Reactor Neutrino Experiment
}

\author{
Liangjian Wen* \\ Institute of High Energy Physics \\ E-mail: wenljeihep.ac.cn
}

\section{On Behalf of the Daya Bay Collaboration}

The neutrino mixing angle $\theta_{13}$ is the gateway of studying CP violation in lepton sectors and determines the trend of future neutrino experiments. The Daya Bay Reactor Neutrino Experiment aims to precisely determine $\theta_{13}$, with the designed sensitivity better than 0.01 in $\sin ^{2} 2 \theta_{13}$ at the $90 \%$ C.L. The experiment takes a near-far relative measurement by comparing the observed electronantineutrino rates and spectra at various baselines from the reactors. Functionally identical antineutrino detectors are deployed in water pools underground, in order to minimize the systematic errors and to suppress the cosmogenic backgrounds. The experiment started physics data taking on Dec. 24, 2011. With 55 days of data, the Daya Bay experiment observed a non-zero value of $\theta_{13}$ at 5.2 standard deviations. Now the Daya Bay experiment has improved the measurement of $\theta_{13}$ using 139 days of data. In this talk, an overview of the experiment and the improved measurement of $\theta_{13}$ are presented. The ratio of the observed to the expected number of antineutrinos assuming no oscillations at the far hall has been measured to be $0.944 \pm 0.007$ (stat.) \pm 0.003 (syst.). In a three-neutrino framework, the $\sin ^{2} 2 \theta_{13}$ is determined to be $0.089 \pm 0.010$ (stat.) \pm 0.005 (syst.), by an analysis of the relative anti-neutrino rates in six detectors.

36th International Conference on High Energy Physics,

July 4-11, 2012

Melbourne, Australia

${ }^{*}$ Speaker. 


\section{Introduction}

Neutrino oscillation is a quantum phenomenon if the mass and flavor eigenstates of neutrinos are mixed. The transformation from mass to flavor eigenstates can be described as a mixing matrix, usually referred to as the PMNS matrix [ [ [ ]], which can be described by three mixing angles $\left(\theta_{12}, \theta_{23}, \theta_{13}\right)$ and a CP violating phase. Neutrino oscillation also depends on the difference of the squared neutrino masses. In the last decade, the three mixing angles and three squared-mass differences have been measured except for $\theta_{13}$ [B]]. First proposed in [四], the sensitivity of neutrino oscillation due to $\theta_{13}$ can be greatly improved if using the near-far detectors near a reactor. This concept has been well utilized by later accelerator and reactor neutrino experiments. By the end of 2011, several hints for non-zero $\theta_{13}$ were reported by T2K [ [5], MINOS [G] and Double Chooz [ [ $]$ ], with significances of $1.7 \sigma$ to $2.5 \sigma$. Meanwhile, a global neutrino data analysis showed $>3 \sigma$ significance for non-zero $\theta_{13}$ [[]].

\section{The Daya Bay Experiment}

The Daya Bay experiment is located at the southern coast of China, $55 \mathrm{~km}$ to Hong Kong and $45 \mathrm{~km}$ to Shenzhen. A detailed description of the Daya Bay experiment can be found in [Q⿴囗十) [0]. The experiment was designed to precisely measure $\theta_{13}$ with a sensitivity of 0.01 or better in $\sin ^{2} 2 \theta_{13}$ at $90 \%$ C.L. The experiment has several key features to achieve such precision mea-

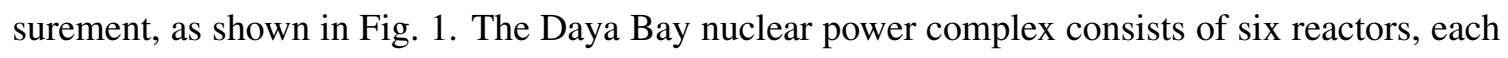
with a maximum of $2.9 \mathrm{GW}$ thermal power, grouped into three pairs. The experiment performs a near-far relative measurement to minimize reactor-related systematic uncertainties. Three experimental halls (EHs) are connected with horizontal tunnels. The Daya Bay near and Ling Ao near halls monitor the neutrino flux from rectors and the far hall measures the oscillation signal. The locations of experimental halls are optimized, taking into account the maximum oscillation baseline, the mountain profile that affects the cosmic-ray flux and the subsequent backgrounds in underground detectors, as well as the geological conditions for tunnel and laboratory construction. Detector-related systematic uncertainties are minimized by deploying multiple functional identical detectors at each experimental hall. In addition, the detectors are placing deep underground for good cosmic shielding, therefore to reduce the background-related systematic uncertainties.

Fig. $\square$ illustrates the layout of the Daya Bay detectors in a near hall. In each EH, multiple antineutrino detectors (ADs) sit side by side, allowing cross checks of detector-related uncorrelated systematic uncertainty. The $\bar{v}_{e}$ s are detected via the inverse $\beta$-decay (IBD) reaction, $\bar{v}_{e}+p \rightarrow e^{+}+n$. Each $\mathrm{AD}$ has $20 \mathrm{t}$ of gadolinium-doped liquid scintillator (Gd-LS) as target and $20 \mathrm{t}$ of liquid scintillator (LS) as gamma catcher. The coincidence of the prompt scintillation from $e^{+}$and the delayed neutron capture on Gd provide a distinctive $\bar{v}_{e}$ signature. In each $\mathrm{EH}$, the ADs are immersed in a water pool, which provides good shielding against ambient radiation with $>2.5 \mathrm{~m}$ of high-purity water in all directions. Each water pool is segmented into inner and out water shields (IWS and OWS) and instrumented with photomultiplier tubes (PMTs) to function as Cherenkov-radiation detectors, for the purpose of tagging cosmic muons and vetoing the cosmogenic backgrounds. Furthermore, an array of resistive plate chambers (RPC) covers on the top, providing a cross check to reduce the uncertainties of muon veto efficiency. 


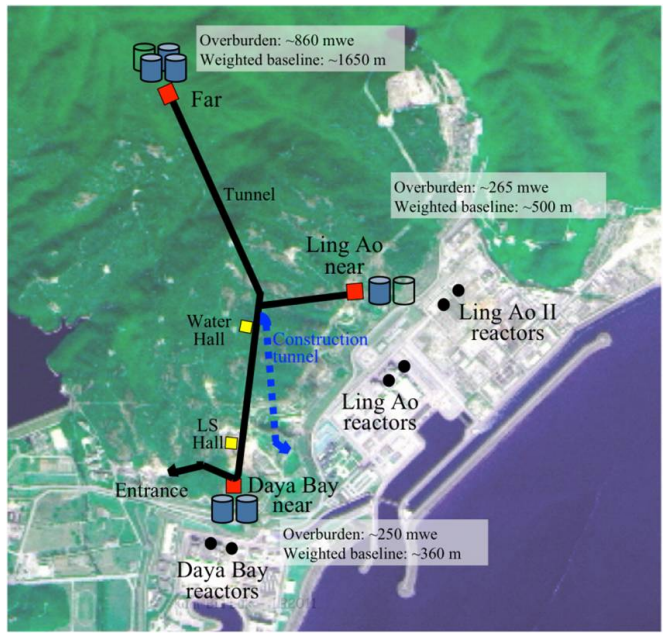

Figure 1: Layout of the Daya Bay experiment.

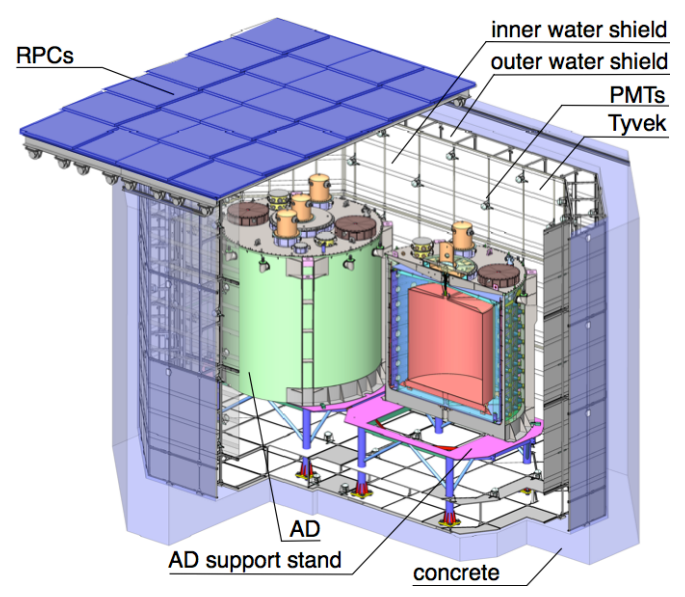

Figure 2: Schematic layout of the Daya Bay detectors in a near hall.

\section{Neutrino Oscillation Analysis}

The detector energy calibration was performed by three automated calibration units (ACUs) mounted on each AD's lid shown in Fig. [1. A light-emitting diode (LED), a ${ }^{68} \mathrm{Ge}$ source and a combined source of ${ }^{241} \mathrm{Am}-{ }^{13} \mathrm{C}$ and ${ }^{60} \mathrm{Co}$ can be remotely deployed into the Gd-LS and LS liquid volumes along three vertical axes. The PMT gains are calibrated by low intensity LEDs. The energy calibration parameter (p.e. per $\mathrm{MeV}$ ) was determined by deploying ${ }^{60} \mathrm{Co}$ source at the detector center. The sources were deployed once per week to correct the weak time dependence. A scan along the vertical axis using ${ }^{60} \mathrm{Co}$ source from each of the three ACUs was used to obtain a common non-uniformity correction function for all the ADs. The neutron energy scale was set by comparing ${ }^{60} \mathrm{Co}$ events with neutron capture on $\mathrm{Gd}$ events from the ${ }^{241} \mathrm{Am}_{-}{ }^{13} \mathrm{C}$ source at the detector center. The energy scale uncertainty was studied by comparing the energy peaks for all types of events in all six ADs, using the neutron capture on Gd from anti-neutrinos and muon spallation products, each of calibration source, and alphas from Polonium decay in the GdLS [U1, [2]. The relative difference falls within a band of $0.5 \%$, quoted as the same uncorrelated uncertainty among ADs, which lead to a $0.12 \%$ relative uncertainty of delayed energy cut efficiency among detectors. Currently we did not preform a nonlinearity correction versus energy.

Anti-neutrino candidates were selected by the following criteria. The energy of the prompt and delayed candidates were required to satisfy $0.7 \mathrm{MeV}<E_{p}<12.0 \mathrm{MeV}$ and $6.0 \mathrm{MeV}<E_{d}<$ $12.0 \mathrm{MeV}$, respectively, and $\Delta t=t_{d}-t_{p}$ must have satisfied a $1<\Delta t<200 \mu s$ coincidence, where $t_{p}$ and $t_{d}$ are the timestamps of the prompt and delayed signals. A multiplicity cut required no additional candidate with $E>0.7 \mathrm{MeV}$ in the interval $200 \mu \mathrm{s}$ before $t_{p}, 200 \mu \mathrm{s}$ after $t_{d}$, or between $t_{p}$ and $t_{d}$. The prompt-delayed pair was vetoed if the delayed candidate satisfied any of the conditions $-2 \mu s<t_{d}-t_{\mu_{\mathrm{WS}}}<600 \mu \mathrm{s}$ (Pool muon), $0<t_{d}-t_{\mu_{\mathrm{AD}}}<1000 \mu \mathrm{s}$ (AD muon), or $0<t_{d}-t_{\mu_{\mathrm{sh}}}<1 \mathrm{~s}$ (AD showering muon). The prompt energy, delayed energy and capture-time distributions for data showed good agreement with MC, respectively.

The absolute efficiencies were predicted by MC. The uncertainties of neutron spill-in (neutrons from IBD interactions outside the target volume were captured by Gd in the target volume) 
efficiency and Gd capture ratio were the two main sources of correlated uncertainties and estimated from the differences between data and MC. For our relative measurement, absolute efficiencies as well as correlated uncertainties effectively canceled. Only uncorrelated uncertainties contribute to the final error. The total uncorrelated uncertainty is $0.2 \%$ [ $\square$, [2] , better than our baseline design $0.38 \%[Q]$. The largest uncorrelated uncertainty is from delayed-energy cut $(0.12 \%)$. The second contribution is from target protons $(0.03 \%)$, achieved by using one batch of target scintillator and precise filling for all detectors. The relative uncertainties were checked by a side-by-side comparison with two near detectors (AD1 and AD2) at Daya Bay near hall [ए0, 2]]. The measured ratio of the total anti-neutrino rates in $\mathrm{AD} 1$ and $\mathrm{AD} 2$ was $0.987 \pm 0.004$ (stat.) \pm 0.003 (syst.), consistent with the expected ratio 0.982 [ए2]].

The largest background in the anti-neutrino candidates are the accidental backgrounds, defined as any pair of otherwise uncorrelated signals that accidentally satisfied the anti-neutrino event selection criteria. The expected background rates were calculated using the rate of prompt-type and delayed-type signals. An alternate method, so-called off-window method, was developed to estimate such background [12]. The background estimation was also validated by comparing the distributions of distance between the reconstructed vertices for the prompt and delayed signals of the antineutrino candidates and accidental coincidences selected by the off-window method. The Background/Signal ratio $(B / S)$ for accidental backgrounds accounted for $4.0 \pm 0.05 \%(1.5 \pm 0.02 \%)$ of the far (near) halls.

The uncertainties in backgrounds were dominated by two sources of correlated signals, the cosmogenic $\beta$-n isotopes ${ }^{9} \mathrm{Li} /{ }^{8} \mathrm{He}$ and the three $0.5 \mathrm{~Hz}{ }^{241} \mathrm{Am}-{ }^{13} \mathrm{C}$ neutron calibration sources inside the ACUs on top of each AD. The ${ }^{9} \mathrm{Li} /{ }^{8} \mathrm{He}$ background was evaluated using a method to fit the distribution of the time since the last muon. By assuming that most of the ${ }^{9} \mathrm{Li} /{ }^{8} \mathrm{He}$ production was accompanied with neutron generation, the $\mathrm{AD}$ tagged muon events with no follow-on neutron were rejected from the muon sample, resulting in an improvement of the fitting precision. The method was extensively discussed in [12]. The $B / S$ for ${ }^{9} \mathrm{Li} /{ }^{8} \mathrm{He}$ backgrounds was estimated to be $0.3 \pm 0.2 \%(0.4 \pm 0.2 \%)$ at the far (near) halls. The formation of Am-C correlated backgrounds was a prompt gamma signal from neutron inelastic scattering on iron, followed by the delayed gamma rays produced by capture on stainless steel. The $B / S$ for Am-C correlated background was estimated to be $0.3 \pm 0.3 \%(0.03 \pm 0.03 \%)$ at the far (near) halls.

Other correlated backgrounds due to energetic neutrons from cosmogenic products (i.e. fast neutrons) and $(\alpha, \mathrm{n})$ nuclear interactions are negligible. The energetic cosmogenic neutrons could mimic anti-neutrino events by recoiling off a proton then being captured on Gd. Such background was estimated by extrapolating the prompt energy distribution between 12 and $100 \mathrm{MeV}$ down to $0.7 \mathrm{MeV}$. Three additional methods were used to provide cross checks and the results were consistent [12]. The $B / S$ for fast neutron backgrounds was estimated to be $0.07 \pm 0.03 \%(0.12 \pm 0.05 \%)$ at the far (near) halls. The backgrounds caused by ${ }^{13} \mathrm{C}(\alpha, n){ }^{16} \mathrm{O}$ reaction was calculated using the measured alpha-decay rates and neutron yield determined by Geant $4 \mathrm{MC}$. The $B / S$ for such backgrounds was estimated to be $0.05 \pm 0.03 \%(0.01 \pm 0.006 \%)$ at the far (near) halls.

The $\bar{v}_{e}$ flux of each reactor was calculated from the simulated fission rate of four main isotopes $\left({ }^{235} \mathrm{U},{ }^{239} \mathrm{Pu},{ }^{238} \mathrm{U}\right.$ and ${ }^{241} \mathrm{Pu}$ ) and the anti-neutrino spectrum per fission (See Ref. [प], ㅁ] ] and references therein). The thermal power measured by the power plant was used for normalization when simulating the fission rate. Our near-far relative measurement was independent of reactor 
flux models, and the uncorrelated reactor uncertainty was estimated to be $0.8 \%$ [ए], ए2].

The $\bar{v}_{e}$ rate in the far hall was predicted with a weighted combination of the two near hall measurements assuming no oscillation. The ratio of the observed to expected rate of all three ADs at the far hall was measured to be $R=0.944 \pm 0.007$ (stat.) \pm 0.003 (syst.). Fig. B] shows the ratio of measured versus expected signals in each detector. In a three-neutrino framework, an analysis of relative anti-neutrino rates of the six detectors determined $\sin ^{2} 2 \theta_{13}=0.089 \pm 0.010$ (stat. $) \pm$ 0.005 (syst.), using a $\chi^{2}$ method with pull terms accounting for the correlation of the systematic errors. The observed $\bar{v}_{e}$ spectrum in the far hall was compared to a prediction based on the near hall measurements. The distortion of the spectra is consistent with that expected due to oscillations at the best-fit $\theta_{13}$ obtained from the rate-based analysis.

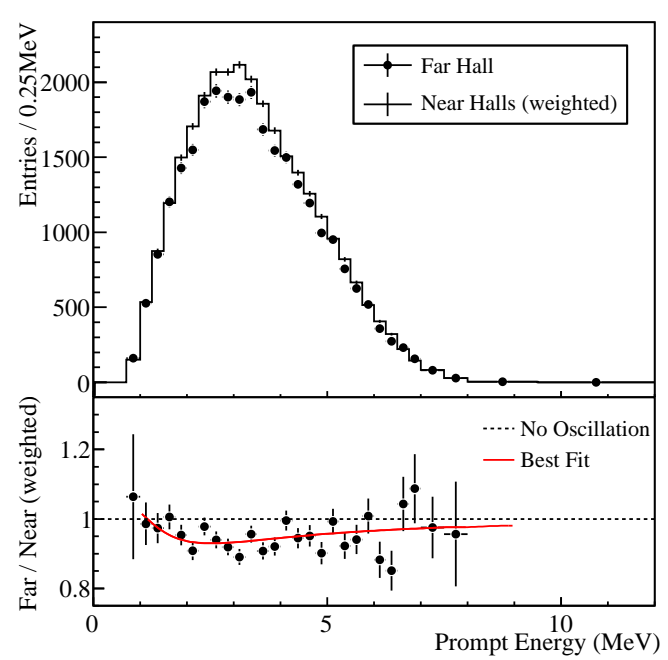

Figure 3: Top: Measured prompt energy spectrum of the far hall (sum of three ADs) compared with the no-oscillation prediction based on the measurements of the two near halls. Bottom: The ratio of measured and predicted nooscillation spectra. The solid curve is the expected ratio with oscillation for $\sin ^{2} 2 \theta_{13}=0.089$.

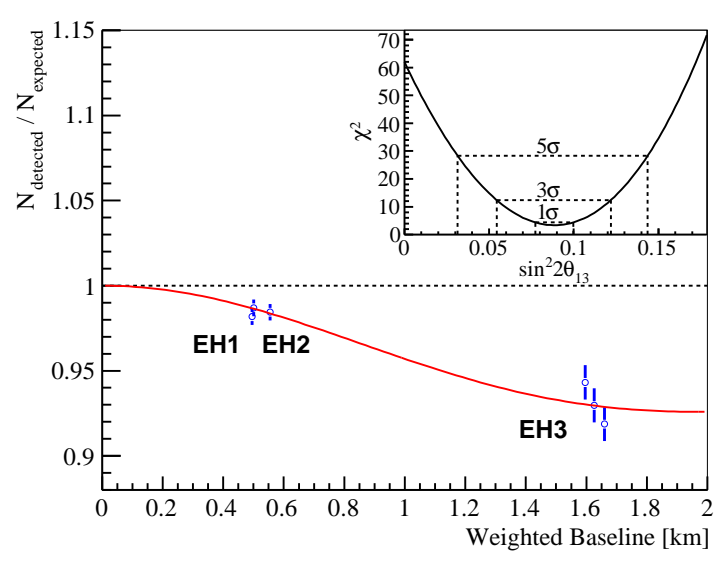

Figure 4: Ratio of measured versus expected (nooscillation) signals in each detector. The oscillation survival probability at the best-fit value is given by the smooth curve. The AD4 and AD6 data points were displaced by -30 and $+30 \mathrm{~m}$ for visual clarity.

\section{Conclusion and Outlook}

Using 139 days of data, the Daya Bay experiment has confirmed the previous observation of reactor electron-antineutrino disappearance and has provided an improved measurement to $\theta_{13}$. In a three-neutrino framework, the disappearance leads to $\sin ^{2} 2 \theta_{13}=0.089 \pm 0.010$ (stat.) \pm 0.005 (syst.). The experiment will take a comprehensive calibration campaign to improve the energy reconstruction for spectral shape analysis. With the last two ADs installed, the experiment will continue to run for at least 3 years to measure $\sin ^{2} 2 \theta_{13}$ to $\sim 5 \%$ precision, by both accumulating more statistics and reducing systematic uncertainties. The high statistics of near halls will also provide the most precise measurement of reactor anti-neutrino flux and spectrum at corresponding baselines. In addition, by measuring the neutrino spectrum distortion, the experiment could measure the effective 
squared-mass difference (a combination of $\Delta m_{31}^{2}$ and $\Delta m_{32}^{2}$ ). The measured large $\theta_{13}$ stimulates the next round of experimental quests to measure mass hierarchy and $\mathrm{CP}$ violation phase.

\section{Acknowledgement}

The Daya Bay experiment is supported in part by the Ministry of Science and Technology of China, the United States Department of Energy, the Chinese Academy of Sciences, the National Natural Science Foundation of China, the Guangdong provincial government, the Shenzhen municipal government, the China Guangdong Nuclear Power Group, Shanghai Laboratory for Particle Physics and Cosmology, the Research Grants Council of the Hong Kong Special Administrative Region of China, University Development Fund of The University of Hong Kong, the MOE program for Research of Excellence at National Taiwan University, National Chiao-Tung University, and NSC fund support from Taiwan, the U.S. National Science Foundation, the Alfred P. Sloan Foundation, the Ministry of Education, Youth and Sports of the Czech Republic, the Czech Science Foundation, and the Joint Institute of Nuclear Research in Dubna, Russia. We thank Yellow River Engineering Consulting Co., Ltd. and China railway 15th Bureau Group Co., Ltd. for building the underground laboratory. We are grateful for the ongoing cooperation from the China Guangdong Nuclear Power Group and China Light \& Power Company.

\section{References}

[1] B. Pontecorvo, Sov. Phys. JETP 6, 429 (1957) and 26, 984 (1968).

[2] Z. Maki, M. Nakagawa, and S. Sakata, Prog. Theor. Phys. 28, 870 (1962).

[3] J. Beringer et al. [Particle Data Group], Phys. Rev. D 86, 010001 (2012)

[4] L. A. Mikaelyan and V. V. Sinev, arXiv:hep-ex/9908047

[5] K. Abe et al. [T2K Collaboration], Phys. Rev. Lett. 107041801 (2011)

[6] P. Adamson et al. [MINOS Collaboration], Phys. Rev. Lett. 107, 181802 (2011)

[7] Y. Abe et al. [Double Chooz Collaboration], Phys. Rev. Lett. 108, 131801 (2012)

[8] G. L. Fogli et al., Phys. Rev. D 84, 053007 (2011)

[9] X. H. Guo et al. (Daya Bay Collaboration), Proposal of the Daya Bay experiment, arXiv:hep-ex/0701029 (2007).

[10] F. P. An et al. (Daya Bay Collaboration), Nucl. Instr. and Meth. A 685, 78 (2012).

[11] F. P. An et al. [Daya Bay Collaboration], Phys. Rev. Lett. 108, 171803 (2012).

[12] F. P. An et al. (Daya Bay Collaboration), arXiv:1210.6327 (2012), to appear in Chinese Physics C. 